\title{
3 Research Square

\section{Assessment of the Implementation of Tuberculosis and Diabetes Care in health systems: A Situational Analysis in Western Nigeria for consideration}

\section{ASAGA MAC PETER ( $\sim$ pasaga123x@gmail.com )}

Albert-Ludwigs-Universitat Freiburg https://orcid.org/0000-0003-0038-8896

JUDE OSAGIE Aighobahi.

Albert-Ludwigs-Universitat Freiburg

\section{Research article}

Keywords: tuberculosis, non-communicable diseases, screening and treatment

Posted Date: August 2nd, 2019

DOI: https://doi.org/10.21203/rs.2.12294/v1

License: (c) (1) This work is licensed under a Creative Commons Attribution 4.0 International License.

Read Full License 


\section{Abstract}

Background: Tuberculosis (TB) coexists with other non-communicable diseases (NCDs), including Diabetes Mellitus (DM). Smoking increases the risk of TB as well as DM. Health systems are poorly prepared in many low middle income countries (LMICs) and are currently facing the "triple burden of smoking, TB, and DM" that drives these countries into the vicious cycle of poverty. Methods: A crosssectional study method was carried out to assess the proportion of TB care centers that included integration measures for diabetic care as well as those providing DM care that included integration measures for TB. A list of 49 health care centers in Lagos offering TB care and managing Diabetes patients were recruited. A focus Group Discussion(FGD) and Individual interviews were conducted to investigate health care providers ' knowledge, attitudes and practices and the barriers encountered in the process of integrating TB and DM care. Results: Out of the 49 health care centres recruited in this study, $6 \%$ of health care units are aware of a surveillance to screen for diabetes in tuberculosis patients, while $2 \%$ of health facilities confirmed awareness of a surveillance to screen for tuberculosis in diabetes patients. $91 \%$ of health centres either verified the lack of or no understanding of monitoring of both diseases. The percentage of health facilities that have existing guideline on TB and DM screening was evaluated, it was perceived that $8 \%$ of health facilities had implemented a guideline to screen for DM in TB patients, while $4 \%$ of these Care Centres have implemented a guideline for diabetes patients to be screened for TB. Conclusion TB/DM integrative screening, treatment and management could be better attained if both co-morbidities integration program is initiated in the healthcare centres and policies of western states and Nigeria as a whole.

\section{Background}

Tuberculosis (TB) coexists with other non-communicable diseases (NCDs), including Diabetes Mellitus (DM). Smoking increases the risk of TB as well as DM. Health systems are poorly prepared in many low middle income countries (LMICs) and are currently facing the "triple burden of smoking, TB, and DM" that drives these countries into the vicious cycle of poverty $[1,4]$.

A link between TB and DM has been shown in several literature, and diabetes triples the risk of TB $[2,3,5]$. TB and DM pose a serious threat to people of all ages and their co-infection involves more complications $[6,25,7,8]$, resulting in significant health challenges. The World Health Organization (WHO) and the International Union against Tuberculosis and Lung Disease have long argued for TB and Diabetes care to be integrated in response to their risk $[9,15,10,11,12]$. Despite these recommendations, progress towards the integration of TB and DM care is largely unknown or not taken into consideration. With a large TB burden and growing DM cases, Nigeria and many other LMICs have little or no data available $[13,71,20,14]$. In this study, we evaluate the following goals: the level of integration of T B and DM care and its contribution to the overall well-being of people living with both morbidities; the proportion of TB care services in Nigeria (Lagos) that included diabetic integration care measures as well as those providing DM care that included TB integration measures. We are also investigating the knowledge, 
attitudes and practices of health care providers and barriers to integrating TB and DM care in the state of Lagos, Nigeria.

\section{Methods}

\section{Study Area}

This study was carried out in Lagos state, Nigeria, the most populated city in West Africa with a population of over 21 million inhabitants consisting of different levels of health centres which include among others primary health care centres, the General hospitals, the federal medical centres, military and navy hospitals, private hospitals and the teaching hospitals. About $50 \%$ of the targeted Health centres providing TB and DM care in Lagos state were studied. The others were not approached due to road accessibility and poor response. Lagos state health centres and general hospitals are governed by the Lagos state health service commission.

A cross-sectional study method was carried out to assess the proportion of TB care centers that included integration measures for diabetic care as well as those providing DM care that included integration measures for TB. A list of 49 health care centers in Lagos offering TB care and managing Diabetes patients were recruited.

\section{Qualitative/Quantitative methods}

A focus Group Discussion(FGD) and Individual interviews were conducted to investigate health care providers ' knowledge, attitudes and practices and the barriers encountered in the process of integrating TB and DM care. Health workers were interviewed on how they felt and responded to TB and DM integration as well as the barriers they face during the implementation of this program. A purposive sampling questionnaire, based on the accessibility and availability of health care facilities was administered. The survey questionnaire was designed in accordance with the WHO guidelines [25] and regulations as well as the BALI declaration, keeping in view the collaborative framework and the screening and management of TB/DM. The main areas were: Socio-demographic characteristics, Clinical practice profile e.g., type of practice (public or private), number of health workers, estimated number of TB and DM patients seen per month, Questions on tuberculosis and diabetes care (screening and management measures) and Questions on TB-DM integration (collaborative, screening activities and management).

\section{Sample size}

A total number of 100 health centres were identified in Lagos. A response rate of 50-80 were expected with an estimate of $50 \%$ of health centres with integration measures and an acceptable margin of error of $5 \%$. 
Figure 1: Geographical locations of health centres in Lagos; with the Dots representing the location of health centres approached and included in the TB-DM study.

\section{Statistical Analysis}

Responses to the closed questions were entered into the computer and anlysed with SPSS. . Responses to open ended questions were transcribed and analysed using categorical evaluation. Proportions were calculated and STATA was used to calculate the $95 \%$ Confidence intervals of integrative measures of screening for TB among DM patients, screening for Diabetes among TB patients, as well as TB, HIV and DM screening of all health centres.

\section{Results}

\section{Table 1: Characteristics and analysis of health centres in the study area}

A total of 49 health facilities were recruited in the TB-DM study survey; $38 \%$ had $5-10$ nurses, $28 \%$ had more than 10 nurses working in the facility, while $32 \%$ of health centers did not respond.

Most health centers (28.5\%) had less than 5 doctors, 7(14.2\%) health centers had between 5-10 doctors, $24.5 \%$ had more than 10 doctors and $16(32.6 \%)$ health centers did not response to the questions. In administrative department, $9(18.3 \%)$ facilities employed less than 5 workers, $14.2 \%$ of health facilities harbour 5-10 administrative workers while more than 10 employees worked in $26.5 \%$ of the health facilities.

The total number of patients seen by the health facilities in a month was investigated. It was discovered that more health facilities 29(59.1\%) witnessed between 100-500 patients per month, 4(8.16) facilities saw less than 100 patients in a month while more than 500 patients visited $24.5 \%$ of the health facilities included in this survey. Most of the health facilities studied during this survey 19(38\%) attested to seeing more than 50 cases of DM patients in a month, $7(14.2 \%)$ of the facilities witnessed less than $10 \mathrm{DM}$ patients in a month, while 16(32.6) health facilities confirmed to have seen between 10-50 patients in a month. There was no response from 7 health facilities. Finally, the total number of TB patients seen in a month was investigated, 18(36.7\%) witnessed between 10-50 patients reporting with TB, while 4(8.16\%) facilities saw more than 50 patients with TB in a month. However, less than 10 patients visited $15(30.6 \%)$ of the health facilities included in this survey. 
Most of the health centres plotted amid this overview confirmed 19(38\%) adjure seeing in excess of 50 cases of DM patients in a month, $7(14.2 \%)$ of the centres saw under $10 \mathrm{DM}$ patients in a month, while $16(32.6 \%)$ asservate to have seen between $10-50$ patients in a month. Seven health facilities did not respond

In closing, the total number of TB patients observed in a month was scrutinized, 18(36.7 \%) witnessed between 10-50 TB patients, while $4(8.16 \%)$ facilities saw more than 50 TB patients in a month. Less than 10 patients, however, visited $15(30.6 \%)$ of the health facilities included in this study survey.

\section{Table 2: Referrers, Medical diagnostics and Care of TB, HIV and DM in health facilities}

Table 2 demonstrates that 30(61.2\%) TB cases were treated in their facilities while 19(38.8\%) allude patients to different centres. In the case of DM 38(77.6\%) of health centres, offered DM management, while $11(22.4 \%)$ were allude to other centres. $41(84 \%)$ offered treatment for HIV patients and 8(16\%) were referred to other facilities. 37(76\%) dependably suggested HIV screening for TB patients, 3(6.25\%) requested that the HIV test be based on risk factors and one health center negated. 43(87.7\%) offered HIV testing and counseling, 3(6.12 \%) offered HIV testing, but counseling was done elsewhere. 2(4\%) referred patients to do the test and counselling at other centres. There was however, no response from one of the centres.

\section{Table 3: Implementation and integration of TB and DM in Health Centres in Lagos offering only TB treatment.}

In Table 3, $6 \%$ of health centres recruited for this study acknowledged that there was a surveillance of diabetes care actuated among patients with tuberculosis in Lagos state. $39 \%$ of the health centres had no proposition on the existence of a surveillance, $55 \%$ of the centres were hesitant if there was a surveillance of DM among TB patients.

There was a guideline for screening all tuberculosis patients for diabetes in $4(8 \%)$ health centers, whereas 27 (55\%) health centers did not enforce the guidelines; there were instances of uncertainty in 18 $(37 \%)$ health centers as the different health centers were not sure of such rules. $25(51 \%)$ of the health employees included in this study were not familiar with routine diabetes screening in patients diagnosed with TB. However, 24 (49\%) verified diabetes screening in patients with tuberculosis at different moments, sometimes screened $45 \%$, most screened $2 \%$, and always screened $2 \%$. Although 29 (59\%) said that in TB patients they almost never screened for diabetes, 20 (41\%) of them agreed to screened at times. From this research, it was found that $14(28.57 \%)$ screened at different moments, 4 (8.16\%) screened at diagnosis and begin of therapy, $2.04 \%$ screened during therapy, while $59 \%$ did not answer the question. In all instances, 20 (40.8\%) screening was performed for patients with diabetes risk variables (e.g. obesity, gestational diabetes or family diabetes), whereas 29 (59.18\%) did not answer the question. 
During the research, it was contrived that screening for diabetes in patients with TB was not performed in $20(40 \%)$ of instances because they think it is not a significant co-morbidity in the nation. $10 \%$ attested to have received information on diabetes from the treating physician. Some of the health care centers (12\%) attributed the absence of screening to the absence of medical employees, economic and logistical means, $4 \%$ had no understanding of reasons for not screening, while $32 \%$ did not answer this question. $36 \%$ of health care employees had information about patients with or without tuberculosis who were either diagnosed with diabetes, $26.5 \%$ had information and less than $10 \%$ of patients with or without the illness had no information

\section{Table 4: Implementation and integration of DM and TB in Health Centres in Lagos offering only DM treatment.}

Table 4 showed that $18(36.7 \%)$ of the health centers visited thought that there was no TB monitoring program among patients with diabetes, and $30(61.7 \%)$ were uncertain as to whether this program existed. $1(2 \%)$ of the recruited health centres reported recognized tuberculosis screening monitoring in patients with diabetes. On the other side, the guidelines for screening tuberculosis patient for diabetes had not been enacted by 28 (57.1\%) health centres while the screening was suggested by $2(4 \%) .19$ (38.7\%) health centres were not sure if there were guidelines for screening TB patients for DM., 26 (53\%) of the health workers profiled in this study acknowledged never to have screened for in diagnosed diabetes patients. However, $23(46.8 \%)$ of the centers verified tuberculosis screening in DM patients at distinct times; $4 \%$ always, $6.1 \%$ most times, and $36.7 \%$ do so sometimes. 28 (57\%) facility centres never screened for tuberculosis in patients with diabetes, 20 (40.8\%) sometimes agreed to have screened, no reply was given by $2 \%, 14(28.5 \%)$ tested at different moments, $5(8.16 \%)$ screened at diagnosis and begin of therapy, while $61.2 \%$ did not table this question. Screening was conducted at various instances in patients with risk variables of the disease (e.g. exposure, HIV, alcohol, smoking) $14(28.5 \%$ ) of health care staff screened for diabetes while $5(8.16 \%)$ screened for patients with suggestive symptoms of TB.

Most health centers used the chest x-ray technique for TB screening 18 (35.3\%), while others used gene xpert 13 (25.5\%), sputum Microscopy / Culture/ Sensitivity 12 (23.5\%) and clinical symptom score 8 (15.7\%). It was observed that health workers did not screen for TB in patients with diabetes in 46.9 percent of instances because they thought it was not a significant co-morbidity, $6 \%$ obtained tuberculosis information from the treating Physician, while $6 \%$ attributed the absence of screening to the absence of personnel, economic and logistical resources, $2 \%$ had no screening discernment for the objective, while $34.7 \%$ did not respond. Lastly, $30 \%$ of health workers had information about diabetes patients who are either diagnosed with tuberculosis or not. $26.5 \%$ of these health care staff had information on patient with or without the disease. For about $10-50 \%$ of patients, $4 \%$ of these health care centres had data on these non-communicable diseases. 
Results of Interviews conducted at five health centres that have integrated TB/DM Screening Measures

Five (5) of the 49 participating health centres interviewed, one (1) attested to have integrated screening measures for TB/DM in the facility and identified possible challenges faced during treatment of both diseases. The summary of the interviews excerpts are reported below:

Participants (Head of Centres) in this study stated that the success of TB/DM integration was impeded and encumbered by the inconsistency in the organisation of health care chain. Subjects (Heads of centres) in this study believed that sparse funds were being pervaded in the integration of TB/DM. Findings in this research disclosed that in curbing the threat of this co-morbidity there were problems, according to two the health centres;

"The problem we face usually is about logistics and finance and the education of the populace at large because the orientation is absent and not well conducted in instances in this part of the world" Interviewee 1 Question 2

According to other participating centres, there is a level of acceptability for integrative TB/DM screening as this will go a long way in improving the health care policy of the government. "The people in my opinion think it's the way forward, and it gives them a better way of living and accessing good healthcare. I think the response has been very positive" Interviewee 1 Question 3

Results of interviews for centres that have not integrated TB/DM Screening Measures

Some of the recruited health centres that have not integrated the screening measures of TB/DM identified reasons why integration of TB/DM does not take place in their facility; they alluded however, the importance of screening for both diseases. Below is a detailed explanation of the retrieved information from the participants (Centres).

"Tuberculosis and diabetes are not diseases that we are aware that could be co-infected with each other that is the reason we have not integrated it in our facility" Interviewee 5 Question 1

Lack of awareness and government poor policies on health matters are major setback why integration of TB/DM is hindered in a lot of health care facilities in Nigeria. Many hospitals/health care facilities have 
no idea that there is an on-going collaborative framework on the screening for TB/DM. Hence, they are unaware of guidelines requesting them to screen for these diseases.

"We have not particularly been on the lookout for such association because we haven't really seen any indicators that may make us be on the lookout for that" Interviewee 4 Question 1

Most health care workers in different centres had no prior understanding of TB / DM inclusion, resulting in no needed screening measures being introduced.

Head of health centres believed that integrative testing and therapy for TB / DM would enhance the quality of life of patients as well as rural and urban communities.

"In my opinion I think it would be really important because if something like that is happening and we do not screen people for it that means that we are overlooking something that is so important" Interviewee 5 Question 2

The respondents believed that screening for TB / DM is viable (conveniently performed) since most health care centres have the manpower and equipment available. "Feasibility is not an issue with us. I mean we have the staff, we have the technical abilities, we have the equipment to use, we do all of the TB tests that are necessary. We have the chest $x$-ray, we do our acid fast bacilli, we do that 2-3 times as necessary, we do the gene expert as necessary. So screening for TB at diagnosing of diabetes mellitus should not be a problem for us" Interviewee 4 Question 3

The national tuberculosis control program of the Nigerian government, stop TB and other Nongovernmental Organisations should create an enabling platform /environment for the successful implementation of TB/DM integrative screening and treatment in various primary health care centres in Lagos and across the country.

"I think the government should be responsible for implementing the program because when the government becomes responsible, they are able to put in resources to ensure that adequate awareness is done amongst the people" Interviewee 5 Question 4

\section{Discussion}

Many low- and middle-income nations (LMICs) face both a persistent infectious disease burden and an evolving chronic non-communicable disease (NCD) burden. Tuberculosis and diabetes are driving these 
nations into the vicious cycle of poverty, death and other co-morbidities.

This study assessed the level at which integration of care for tuberculosis and diabetes is carried out in Lagos, Nigeria. It explored the challenges faced by health workers and centres in western Nigeria.

Out of the 49 health care centres recruited in this study, $6 \%$ of health care units are aware of a surveillance to screen for diabetes in tuberculosis patients, while $2 \%$ of health facilities confirmed awareness of a surveillance to screen for tuberculosis in diabetes patients. $91 \%$ of health centres either verified the lack of or no understanding of monitoring of both diseases. These findings are in consonance with the study of [Riza et al., 15] where the low awareness of TB-DM comorbidity was established. These findings could be alluded to poor education, government policies, medical health testing for both morbidities and inadequate tools and staff.

The percentage of health facilities that have existing guideline on TB and DM screening was evaluated, it was perceived that $8 \%$ of health facilities had implemented a guideline to screen for DM in TB patients, while $4 \%$ of these Care Centres have implemented a guideline for diabetes patients to be screened for TB. Further studies were conducted to find out how many health centers regularly screened for TB and diabetes. Findings in this research show that no health centre regularly screen for tuberculosis in patients with diabetes, as well as diabetes screening in patients with tuberculosis is regularly performed, thereby supporting the study of $[15,11,17]$, where it has also been reported that all health employees in different centres are never regularly screening for TB / DM. This conclusion could be attested to lack of awareness of the co-morbidity and integration policies of the various centres as well as staff expertise, confrere and acquaintance with this public health emergency.

$41 \%$ of healthcare centres perform diabetes screening on TB patients, while $59 \%$ never perform the screening, $40 \%$ of health care centres tested for TB in DM while $57 \%$ never screen for tuberculosis in patients with DM. This is due to the related risk variables in the acquisition of TB and DM. Unfortunately, our research is the only study that has established these fact(s) in Nigeria. There is no known signature elsewhere in Nigeria about similar findings and fact. The ratiocinate are not inconsistent from the determinants suggested earlier.

Subjects (Health centres) were interviewed regarding knowledge and opinion on the challenges and integration of TB and DM. Of the 5 centres interviewed, 4 had no knowledge on TB/DM integrated services in their health facilities. One of the major findings was lack of awareness on TB/DM co-infection or coexistence. Correspondingly, a study carried out in India by Sharm et al, WHO $[16,25,22]$ also identified lack of awareness as a major element for hindered integration and growth of TB-DM integrated clinical services.

Some healthcare facilities in Lagos State thought there was no link between TB and diabetes, but they opined that both disease management could provide a better platform for patients with TB and DM 
outcomes. Goel, Singh et al [7] advocated a similar policy for better service delivery and treatment outcome if TB and DM are managed concurrently.

In most primary health care centers in most Nigerian cities, the shortage of qualified health workers and staff is a significant challenge. This is the consequence of the health ministries ' absence of engagement, policies and inept attitudes in offering an enabling atmosphere where health workers are trained and retrained to manage these illnesses. Other similar studies reported these findings, Ukwaja et al, Trinidad et al, Theunion $[20,19,18]$. The project's advancement (TB/DM Integration) in a comparable scenario in South India was based on the availability of qualified health laboratory employees and free health facilities $[11,26]$.

\section{Conclusion}

TB/DM integrative screening, treatment and management could be better attained if both co-morbidities integration program is initiated in the healthcare centres and policies of western State and Nigeria as a whole. This is asserted to the high prevalence of TB/DM co-infection that is grossly under-reported, as a result institutions and capacity building should be put in place in fighting the menace of these comorbidities. For better screening, treatment and management of TB/DM, convenient and feasible measures such as integration of TB/DM unit in various healthcare facilities in Lagos, training of staff, building of capacities and networking with various international organisations in the treatment of these comorbidities should be a priority on the table of States and Federal Government Health Ministries in Nigeria. This will help in reducing the co-morbidities and stigmatization associated with these diseases. A similar policy had been initiated in some African countries on how to reduce stigmatization at community levels $[21,23,24]$.

In closing, this study was able to establish that very few healthcare facilities have the understanding, monitoring and integration of the TB/DM screening and management as co-infection and morbidities. Various determinants were attributed to the downward implementation on the management program of these two non-communicable diseases, these elements and components include among others, logistics and finance, lack of awareness, education and problem with acceptance of the co-morbidity. It is also imperative to reverse the lackadaisical attitudinal policies of the Nigerian government on DOTS, and also diabetes management must be made a priority of all rural and primary health care centres in the country. Government as well as the international organisation should show commitment in the area of training and re-training of health care workers in the fight against TB and DM, to avoid/reduce Disability Adjusted Life Years (DALYS) and increase Quality adjusted life years (QALYS).

\section{Strengths of this research study}

This is the only know signature on the survey and assessment of the integration of care of tuberculosis and diabetes by healthcare facilities in western Nigeria and Nigeria. 


\section{Limitations of the study}

This study was conducted in limited number of health centres as time was a major constraint. Research bias was a major limitation in this cross sectional study as very few health centres were recruited out of many. As a result, the findings are not a complete representation of the integration of TB and DM comorbidity in Nigeria. Some of the questions were filled with events of the past; there could be difficulties for the participants to remember the answer to some questions. As a result, there were no responses to some questions. More health centres and tertiary institutions need to be recruited in the 6 geopolitical zones in Nigeria to establish a true picture of TB/DM co-morbidities.

\section{Abbreviations And Acronyms}

CDC: Centres for Disease Control and Prevention

CIDA: Canadian International Development Agency

CSOs: Centre for Social Organization of Schools

DALYs: Disability Adjusted Life Years

DOT: Directly Observed Treatment, Short-course

DM: Diabetes Mellitus

IDF: International Diabetic Federation

ILEP: International Federation of Anti-Leprosy Associations

LGA: Local Government Area

LMICs: Low and Middle Income Countries

MDR-TB: Multi-Drug-Resistant Tuberculosis

NTLCP: National Tuberculosis and Leprosy Programme

PATH: Program for Appropriate Technology in Health

PEPFAR: President's Emergency Plan for AIDS Relief

T2DM: Type 2 Diabetes Mellitus

TB: Tuberculosis 
Th: Helper Cells

USAID: United States Agency for International Development

WHO: World Health Organisation

\section{Declarations}

\section{Ethics approval and consent to participate}

The ethical committee of the University medical centre, Freiburg approved the study; ethical approval No: 144/18. An informed written consent was obtained from study participants before the commencement of the work.

\section{Consent for publication}

$\mathrm{N} / \mathrm{A}$

\section{Availability of data and material}

Data is contain in the manuscript body, except other key information which can be obtain from the author upon request.

\section{Competing interests}

Authors declare that they have no competing interests

\section{Funding}

$\mathrm{N} / \mathrm{A}$

\section{Authors' contributions}

JA: Design and initiated the project, sampling and statistical analysis

PA: Design the project and wrote the manuscript, analysed the results

All authors read and approved the manuscript for submission and publication.

\section{Acknowledgements}

We wish to like to thank Dr Berit Lange for rendering me all the necessary support during the research. We wish to also thank the members of staff at the Biological Anthropology and Zentrum Medizin undGesellschaft (ZMG) at the University of Freiburg. We thank the coordinator of the program Dr Sonia Diaz-Monsalve, for all the advice and support given to us. 


\section{References}

1. Al-Rifai, R., Pearson, F., Critchley, J. and Abu-Raddad, L. (2017). Association between diabetes mellitus and active tuberculosis: A systematic review and meta-analysis. PLOS ONE, 12(11), p.e0187967.

2. Anand, T., Kishore, J., Isaakidis, P., Gupte, H., Kaur, G., Kumari, S., Jha, D. and Grover, S. (2018). Integrating screening for non-communicable diseases and their risk factors in routine tuberculosis care in Delhi, India: A mixed-methods study. PLOS ONE, 13(8), p.e0202256.

3. Anon, (2018). [online] Available at: http://www.who.int/tb/post2015_TBstrategy.pdf. [Accessed 4 Sep. 2018].

4. Baker, M., Harries, A., Jeon, C., Hart, J., Kapur, A., Lönnroth, K., Ottmani, S., Goonesekera, S. and Murray, M. (2011). The impact of diabetes on tuberculosis treatment outcomes: A systematic review. BMC Medicine, 9(1).

5. Courtwright, A. and Turner, A. (2010). Tuberculosis and Stigmatization: Pathways and Interventions. Public Health Reports, 125(4_suppl), pp.34-42.

6. Ekeke, N., Ukwaja, K., Chukwu, J., Nwafor, C., Meka, A., Egbagbe, E., Soyinka, F., Alobu, I., Agujiobi, I., Akingbesote, S., Igbinigie, O., Offor, J., Madichie, N., Alphonsus, C., Anyim, M., Mbah, O. and Oshi, D. (2017). Screening for diabetes mellitus among tuberculosis patients in Southern Nigeria: a multicentre implementation study under programme settings. Scientific Reports, 7(1).

7. Goel, S., Singh, A. and Tiwari, Y. (2014). Arrival time pattern and waiting time distribution of patients in the emergency outpatient department of a tertiary level health care institution of North India. Journal of Emergencies, Trauma, and Shock, 7(3), p.160.

8. Jeon, C. and Murray, M. (2008). Correction: Diabetes Mellitus Increases the Risk of Active Tuberculosis: A Systematic Review of 13 Observational Studies. PLoS Medicine, 5(8), p.e181.

9. Jerene, D., Hiruy, N., Jemal, I., Gebrekiros, W., Anteneh, T., Habte, D., Melese, M., Suarez, P. and Sangiwa, G. (2017). The yield and feasibility of integrated screening for TB, diabetes and HIV in four public hospitals in Ethiopia. International Health, 9(2), pp.100-104.

10. Lawson, L., Muc, M., Oladimeji, O., Iweha, C., Opoola, B., Abdurhaman, S., Bimba, J. and Cuevas, L. (2017). Tuberculosis and diabetes in Nigerian patients with and without HIV. International Journal of Infectious Diseases, 61, pp.121-125.

11. Ogbera, A., Kapur, A., Chinenye, S., Fasanmade, O., Uloko, A. and Odeyemi, K. (2014). Undiagnosed diabetes mellitus in tuberculosis: A Lagos report. Indian Journal of Endocrinology and Metabolism, 18(4), p.475.

12. Ogbera, O., Adeyeye, O., Odeniyi, I. and Adeleye, O. (2013). Knowledge of diabetes mellitus in tuberculosis amongst healthcare workers in Nigeria. Indian Journal of Endocrinology and Metabolism, 17(4), p.704.

13. Pablos-Méndez, A., Blustein, J. and Knirsch, C. (1997). The role of diabetes mellitus in the higher prevalence of tuberculosis among Hispanics. American Journal of Public Health, 87(4), pp.574-579. 
14. ReyPineda G, D. (2014). Type 2 Diabetes Mellitus as a Risk Factor for Tuberculosis. Mycobacterial Diseases, 04(02).

15. Riza, A., Pearson, F., Ugarte-Gil, C., Alisjahbana, B., van de Vijver, S., Panduru, N., Hill, P., Ruslami, R., Moore, D., Aarnoutse, R., Critchley, J. and van Crevel, R. (2014). Clinical management of concurrent diabetes and tuberculosis and the implications for patient services. The Lancet Diabetes \& Endocrinology, 2(9), pp.740-753.

16. Sharma, P., Visnegarwala, F. and Tripathi, V. (2014). Burgeoning double burden of tuberculosis and diabetes in India: Magnitude of the problem - Strategies and solutions. Clinical Epidemiology and Global Health, 2(3), pp.107-116.

17. Sullivan, T. and Ben Amor, Y. (2012). The Co-Management of Tuberculosis and Diabetes: Challenges and Opportunities in the Developing World. PLoS Medicine, 9(7), p.e1001269.

18. org. (2018). [online] Available at: https://www.theunion.org/bali-declaration.pdf [Accessed 4 Sep. 2018].

19. Trinidad, R., Brostrom, R., Morello, M., Montgomery, D., Thein, C., Gajitos, M., Heetderks, A. and Chorba, T. (2018). Tuberculosis screening at a diabetes clinic in the Republic of the Marshall Islands.

20. Ukwaja, Kingsley \& Onyedum, Cajetan. (2013). The performance of a tuberculosis control program in a high burden country: The Nigeria situation. Annals of Tropical Medicine and Public Health. 6. 500502. Accessed on 5 Sep. 2018

21. Welcome, M. (2011). The Nigerian health care system: Need for integrating adequate medical intelligence and surveillance systems. Journal of Pharmacy and Bioallied Sciences, 3(4), p.470.

22. int. (2015). [online] Available at:

http://www.who.int/tb/publications/global_report/high_tb_burdencountrylists2016-2020.pdf [Accessed 22 Jun. 2018].

23. int. (2016). [online] Available at: $\underline{\text { http://www.who.int/tb/publications/diabetes_tb.pdf [Accessed } 5}$ Jul. 2018].

24. Workneh, M., Bjune, G. and Yimer, S. (2016). Assessment of health system challenges and opportunities for possible integration of diabetes mellitus and tuberculosis services in South-Eastern Amhara Region, Ethiopia: a qualitative study. BMC Health Services Research, 16(1).

25. World Health Organization. (2017). Diabetes. [online] Available at: http://www.who.int/en/newsroom/fact-sheets/detail/diabetes [Accessed 28 Jun. 2018].

26. World Health Organization. (2017). Tuberculosis (TB). [online] Available at: http://www.who.int/news-room/fact-sheets/detail/tuberculosis [Accessed 23 Jun. 2018].

\section{Tables}

Due to technical limitations, tables are only available as a download in the supplemental files section.

\section{Figures}




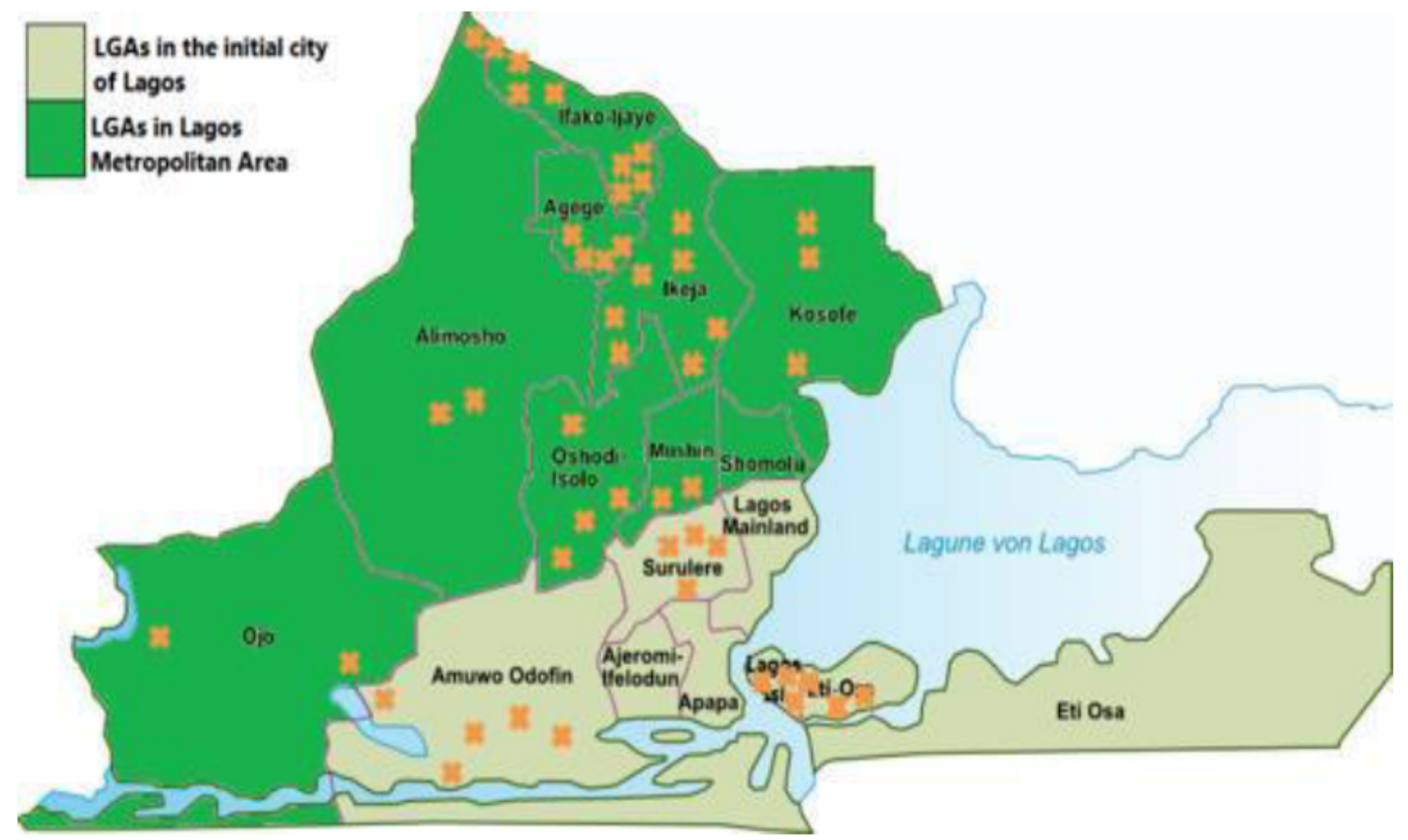

Figure 1: Geographical locations of health centres in Lagos; with the Dots representing the location of health centres approached and included in the TB-DM study.

\section{Figure 1}

Geographical locations of health centres in Lagos; with the Dots representing the location of health centres approached and included in the TB-DM study.

\section{Supplementary Files}

This is a list of supplementary files associated with this preprint. Click to download.

- Tables.pdf 